\title{
Biomarcadores moleculares em câncer: implicações para a pesquisa epidemiológica e a saúde pública
}

\author{
Molecular biomarkers in cancer: implications \\ for epidemiological research and public health
}

Victor Wünsch Filho 1

Gilka J. Figaro Gattás 2

\footnotetext{
${ }^{1}$ Departamento de Epidemiologia, Faculdade de Saúde Pública, Universidade de São Paulo. Av. Dr. Arnaldo 715, São Paulo, $S P$ 01246-904, Brasil. wunsch@usp.br

2 Departamento de Medicina Legal, Ética Médica e Medicina Social e do Trabalho, Faculdade de Medicina, Universidade de São Paulo. Rua Teodoro Sampaio 115 , São Paulo, SP 05405-000, Brasil. gfgattas@usp.br
}

\begin{abstract}
Identification of molecular biomarkers is a common result of current cancer epidemiological research. Both genetic and molecular epidemiology have enjoyed impressive developments in recent decades, with important repercussions on traditional epidemiological approaches. In this paper we evaluate the new frontiers of cancer epidemiology, incorporating both genetic and molecular biology approaches. We examine the current knowledge of molecular biomarkers for exposure and susceptibility to cancer, the role of gene mutations in carcinogenesis, and their application to epidemiological studies. By exploring the status of relevant biomarkers, these approaches become effective in evaluating exposure and susceptibility and show enormous potential for elucidating mechanisms of carcinogenesis and the effect of risk factors in cancer. However, these approaches are necessarily more invasive and raise several ethical issues for consideration by both researchers in public health and society as a whole.
\end{abstract}

Key words Cancer; Genetics; Molecular Biology; Molecular Epidemiology; Epidemiologic Methods

Resumo O desenvolvimento das áreas de genética e biologia molecular tem sido admirável nas últimas décadas e isso tem repercutido intensamente na epidemiologia. Neste artigo, discute-se a ampliação das fronteiras da pesquisa epidemiológica em câncer com a incorporação das técnicas da genética e da biologia molecular. Examina-se o conhecimento atual dos biomarcadores de exposição e de suscetibilidade, o papel das mutações genéticas na carcinogênese, a aplicação destas nos estudos epidemiológicos e implicações para a prevenção. Perscrutando o meio interno dos indivíduos, a epidemiologia molecular e a genética representam um avanço tanto para $a$ avaliação da exposição, quanto para a detecção de indivíduos suscetíveis, e possuem imenso potencial para ampliar a compreensão dos mecanismos da carcinogênese e dos efeitos de fatores de risco no câncer. Entretanto, por serem necessariamente mais invasivas, essas abordagens remetem a importantes questões no campo da ética. A comunidade científica de saúde pública e a sociedade devem guardar vigilância sobre os usos e aplicações deste novo conhecimento, avaliando seus desdobramentos à luz da bioética.

Palavras-chave Câncer; Genética; Biologia Molecular; Epidemiologia Molecular; Métodos Epidemiológicos 


\section{Introdução}

O objetivo da epidemiologia é identificar populações sob risco de desenvolver doenças e propor ações de prevenção e controle. O processo de investigação epidemiológica requer a definição de medidas quantitativas de exposição, bem como da doença estudada e o uso de algum método de associação dessas variáveis. Para a caracterização de populações com maior risco de câncer, a epidemiologia deve operar duas estratégias: obter evidências de exposição a agentes cancerígenos na população e detectar indivíduos com maior suscetibilidade quando expostos a esses agentes, seja por fatores herdados ou adquiridos. A compreensão da etiologia dos tumores malignos, ainda desconhecida apesar dos avanços realizados na pesquisa em câncer nas últimas décadas, provavelmente terá como base o melhor conhecimento das interações entre as diversas exposições ambientais e os fatores genéticos ligados à suscetibilidade individual (Vineis, 1998).

O câncer é considerado uma doença genética que ocorre por um acúmulo de mutações não esperadas por recombinação mendeliana, em virtude, principalmente, de exposição a agentes genotóxicos do meio ambiente (Vogelstein \& Kinzler, 1998). Virtualmente, todos os tumores têm anormalidades gênicas ou cromossômicas. A célula cancerosa passa para sua progênie mensagens sobre o comportamento celular maligno e é este fato que caracteriza o câncer como uma doença letal (Mulvihill \& Tulinius, 1987).

O enorme progresso realizado na área da biologia molecular nas décadas recentes materializa-se atualmente no Projeto do Genoma Humano, uma proposta cooperativa entre centros de pesquisas da América do Norte, Europa e Japão, cujo objetivo é determinar a seqüência completa do DNA do genoma humano (Ellsworth et al., 1997). Em dezembro de 1999, foi anunciado a primeira seqüência completa de um cromossomo humano - o cromossomo 22 (Dunham et al., 1999). Em São Paulo, a Fundação de Amparo à Pesquisa no Estado de São Paulo (FAPESP), juntamente com o Instituto Ludwig de Pesquisa sobre Câncer, está desenvolvendo o Projeto Genoma Humano do Câncer, com a intenção de gerar seqüências de genes humanos envolvidas nesse processo, tendo como base a análise do RNA de certos tumores, e, assim, contribuir com o esforço mundial para a determinação da estrutura completa do genoma humano (Simpson, 1999). Esses avanços têm profundas implicações na pesquisa epidemiológica e deverão acentuar-se no decorrer do século XXI.
Grande parte do conhecimento acumulado no século XX sobre a etiologia das doenças não transmissíveis e sua prevenção pode ser creditada ao aprimoramento dos estudos epidemiológicos para avaliar a exposição de grupos populacionais a fatores de risco ambientais. $\mathrm{O}$ desenvolvimento de modelos estatísticos aplicados à epidemiologia foi essencial nesse processo, entretanto, gradativamente, a abordagem da epidemiologia dos fatores de risco (Silva, 1990) atinge seus limites, mais diretamente ligados à causalidade do que propriamente à formulação de hipóteses etiológicas. Após a identificação de fatores ambientais com riscos relativos expressivos, os epidemiologistas enfrentam o impasse de riscos relativos tênues e de difícil interpretação (Wynder, 1990). A primeira reação para vencer tal obstáculo foi aprofundar os procedimentos de análise, através de refinadas técnicas estatísticas computadorizadas, mas que, em geral, produzem ganhos de apenas poucos décimos no risco relativo (Kuller, 1999). Recentemente, os conceitos da biologia e da genética molecular, assim como novas técnicas laboratoriais, vêm-se integrando aos métodos epidemiológicos (Ellsworth et al., 1997). Apesar das dificuldades inerentes à reaproximação de áreas há muito distantes e com trajetórias independentes, este movimento capitaliza as vantagens de integrar perspectivas e experiências específicas, o que poderá ampliar substancialmente a compreensão dos determinantes epidemiológicos responsáveis pela variação da distribuição de doenças nos indivíduos e nas populações.

A proposta deste trabalho é examinar como o conhecimento da biologia molecular tem sido aplicado nos estudos epidemiológicos, identificar os principais biomarcadores investigados na sua relação com câncer, analisar as dificuldades da integração dessas disciplinas, particularmente do ponto de vista dos epidemiologistas, e analisar os desdobramentos éticos que advirão com a expansão do conhecimento da doença no plano molecular.

\section{Mecanismos da carcinogênese}

As neoplasias caracterizam-se por longos períodos de latência, às vezes tão longos quanto cinco ou seis décadas. Experimentos com animais conduziram a um modelo que propõe a divisão da latência em, pelo menos, dois estágios: iniciação, no qual o agente cancerígeno induz mutações e altera a velocidade da divisão celular, e promoção, estágio sucessivo em que o processo evolui até constituir-se num tu- 
mor observável. Neste processo, a célula se modifica e, tornando-se cancerosa, expande-se com desenvolvimento anômalo formando uma colônia de descendentes denominada clone (Squire et al., 1998).

Evidências epidemiológicas e genéticas sugerem um processo de multiestágios na carcinogênese (Pitot, 1996). Os modelos epidemiológicos procuram associar o tipo de carcinógeno e sua ação em diferentes estágios da carcinogênese com fatores individuais, como a idade e a dose e duração da exposição (Day \& Brown, 1980; Vainio et al., 1992). Os cancerígenos seriam, assim, classificados como agentes que podem afetar estágios precoces ou tardios da carcinogênese. Um carcinógeno atuando em fases precoces da carcinogênese produz efeito retardado, em relação tanto ao aumento da incidência de câncer, seja no início ou mesmo no decorrer da exposição, como à diminuição desta incidência após a exposição ter cessado. De forma análoga, quando um estágio tardio da proliferação celular é modificado pela ação de determinado carcinógeno, as respostas, tanto ao início, quanto à cessação da exposição, são mais rápidas (Pitot, 1996). Na prática, entretanto, a dificuldade em mensurar a exposição e determinar a dose do carcinógeno que atinge o tecido alvo pode dificultar tais interpretações (Vainio et al., 1992).

\section{Genética e câncer}

Incorporando conhecimentos da biologia molecular e da genética, a epidemiologia mantém seus objetivos originais, porém adquire força para obter uma melhor compreensão da etiologia e da patogênese de doenças, subsidiando, assim, mais efetivamente, o planejamento de estratégias de prevenção (Ellsworth et al., 1997; Khoury, 1998). No final dos anos 70, a epidemiologia genética emergiu como disciplina formal (Khoury, 1998). Nos anos 80, outro campo delineou-se na epidemiologia - a epidemiologia molecular (Schulte \& Perera, 1993).

A concentração familial de casos de câncer tem sido descrita como fator de risco para câncer (Browson et al., 1997; Hemminki \& Vaittinen, 1998; Tokuhata \& Lilienfeld, 1963; Wünsch Filho et al., 1995). Esse risco, sugere-se, poderia ser decorrente de mutações herdadas em genes promotores ou supressores de câncer, associadas ou não à capacidade individual de metabolizar substâncias potencialmente carcinogênicas. Polimorfismos de enzimas metabolizadoras de substâncias carcinogênicas são geneticamente determinados e é provável que sejam os mecanismos explicativos da variabilidade interindividual de suscetibilidade à exposição a agentes cancerígenos (Harris, 1987).

Pelo método de análise de segregação, foi observado um padrão de herança co-dominante em famílias com câncer de pulmão, com os tumores tendendo a desenvolverem-se em uma idade mais precoce nos membros de famílias com indivíduos afetados pela doença (Sellers et al., 1990). Gauderman \& Morrison (2000), pesquisando indivíduos que morreram de câncer de pulmão e os parentes destes de primeiro-grau, confirmaram esse fato. Os autores estimaram que a proporção de pessoas com câncer de pulmão portadoras de um locus autossômico dominante excede a $90 \%$ para indivíduos com idade abaixo de sessenta anos. A proporção diminui para aproximadamente $10 \%$ dos casos cujo início da doença se deu aos oitenta ou mais anos de idade. Essas observações dão suporte à hipótese da presença de um locus genético de suscetibilidade para o câncer de pulmão.

A identificação molecular de mutações e polimorfismos genéticos traduz-se para a saúde pública como possibilidade de caracterização da suscetibilidade individual ao câncer, podendo no futuro conduzir a novas perspectivas para a prevenção e o diagnóstico precoce, bem como para o aconselhamento genético e o desenvolvimento de terapêutica gênica. Do ponto de vista da pesquisa, representa obter estimativas mais abrangentes dos riscos, embora não signifique a geração de novas hipóteses etiológicas para o câncer (McMichael, 1994).

\section{Métodos da epidemiologia genética e molecular}

Os métodos da epidemiologia genética alicerçam-se, de um lado, nos modelos clássicos da pesquisa genética, com base em associações entre incidência da doença em diferentes grupos étnicos, concordância do evento entre gêmeos monozigóticos quando comparados a dizigóticos, verificação da incidência da doença nos demais membros da família e associação da doença com sintomas observados em síndromes diversas (Wyszynski, 1998). Por outro lado, fundamentam-se nos desenhos epidemiológicos, como estudos transversais, casocontrole e coortes. Khoury (1998) confere particular atenção à utilidade dos estudos casocontrole na pesquisa em epidemiologia genética. Abordagens epidemiológicas não tradicionais têm sido incorporadas crescentemente na pesquisa genética, a exemplo dos estudos res- 
tritos a casos, cujos controles são definidos dentro do grupo de casos (Yang et al., 1999), e dos estudos de casos utilizando parentes como controles (Flanders \& Khoury, 1996).

Técnicas de epidemiologia molecular induzem adaptações nos desenhos epidemiológicos para ajustá-los à logística de coleta de material biológico. Têm sido propostos os estudos de transição, para estabelecer a ligação entre os experimentos laboratoriais e a epidemiologia de base populacional, caracterizar a variabilidade intra e interindividual dos biomarcadores, avaliar a viabilidade do uso de determinado marcador nas condições de pesquisa de campo e otimizar o uso de biomarcadores (Schulte \& Perera, 1993, 1997).

\section{Biomarcadores moleculares}

Qualquer tentativa de sistematizar uma classificação dos biomarcadores moleculares será inevitavelmente simplificada e estará sempre distante de apreender o verdadeiro papel que cada alteração molecular exerce na carcinogênese. Os biomarcadores têm sido categorizados em três tipos principais: de exposição, de suscetibilidade e de resposta. Os biomarcadores de exposição correspondem à expressão de um agente ambiental ou de seus metabólitos no meio interno dos indivíduos. Os de suscetibilidade indicam indivíduos mais ou menos propensos a desenvolver câncer quando expostos a substâncias cancerígenas. Os biomarcadores de efeito ou de resposta indicam alterações presentes em tumores; são tardios e permitem avaliar o prognóstico da doença. Na Figura 1, formula-se a apresentação sucinta desses biomarcadores.

\section{Biomarcadores de exposição}

A compreensão dos mecanismos da carcinogênese depende, pelo menos em parte, da análise laboratorial dos efeitos de um agente particular na molécula de DNA e nas células. Por outro lado, avaliar a contribuição de um agente isolado em estágios particulares da carcinogênese pode ser difícil, pois interações podem ocorrer entre substâncias químicas e outros fatores, tais como vírus, radiação e, possivelmente, componentes endógenos. Portanto, o conhecimento dos mecanismos de carcinogênese será sempre, hipoteticamente, incompleto (Vainio et al., 1992).

O mecanismo pelo qual agentes químicos e seus metabólitos cancerígenos causam mutações genéticas tem sido intensamente investi- gado nas duas últimas décadas. $\mathrm{O}$ vínculo entre químicos e a molécula de DNA se dá pela formação de ligações covalentes denominadas adutos (Toniolo et al., 1997). Grande número de adutos foram identificados, incluindo os formados pelos hidrocarbonetos policíclicos aromáticos (HPA), as aminas aromáticas e os compostos nitrosos. Os adutos HPA-DNA têm sido os mais estudados e, juntamente com os anticorpos desses adutos encontrados no soro humano, constituem-se em úteis marcadores da dose biologicamente efetiva da exposição a esta substância nos locais de trabalho, a exemplo de siderúrgicas, em ambientes poluídos de cidades ou no tabagismo (Perera et al., 1988, 1992; Philips et al., 1988).

A razão de mensurar adutos carcinógenoDNA para avaliar exposição é baseada na suposição de que a formação de adutos possa ser responsável por mutações em genes críticos para a carcinogênese, se o reparo do DNA não ocorrer antes da divisão celular. Esse raciocínio depende de um modelo causal que envolva os adutos, as mutações e a indução de tumor (Wild \& Pisani, 1997). Mutações em genes envolvidos no processo da carcinogênese observadas em tumores de animais geralmente são consistentes com o tipo esperado de aduto no DNA decorrente da exposição a um químico específico (Greenblatt et al., 1994). Contudo, em geral, o nível de adutos no DNA reflete a exposição passada recente e não a mais remota (Montesano, 1990), fato que representa um obstáculo para a compreensão dos mecanismos da carcinogênese química, mas, por hipótese, indivíduos expostos cronicamente têm maior probabilidade de falhas nos mecanismos de reparo do DNA. Todavia, não há, ainda, evidências experimentais da seqüência: formação de adutos, mutação e ocorrência de tumor (McMichael, 1994).

Os adutos carcinógeno-DNA podem ser eliminados por sua instabilidade química e pela ação de enzimas responsáveis nos processos de reparo do DNA (Skipper \& Tannenbaum, 1990). A variabilidade interindividual da suscetibilidade humana aos cancerígenos depende não apenas do estado nutricional e de saúde do hospedeiro, incluindo doenças pré-existentes, mas fundamentalmente da capacidade de reparo do DNA. Esse mecanismo tem intrínseca relação, também, com a competência genética individual de síntese de enzimas ativadoras ou de detoxificação de substâncias cancerígenas (Vainio et al., 1992).

A identificação a priori do risco aumentado para o desenvolvimento de câncer pode ser feita, ainda, mediante testes de mutagenicidade, 


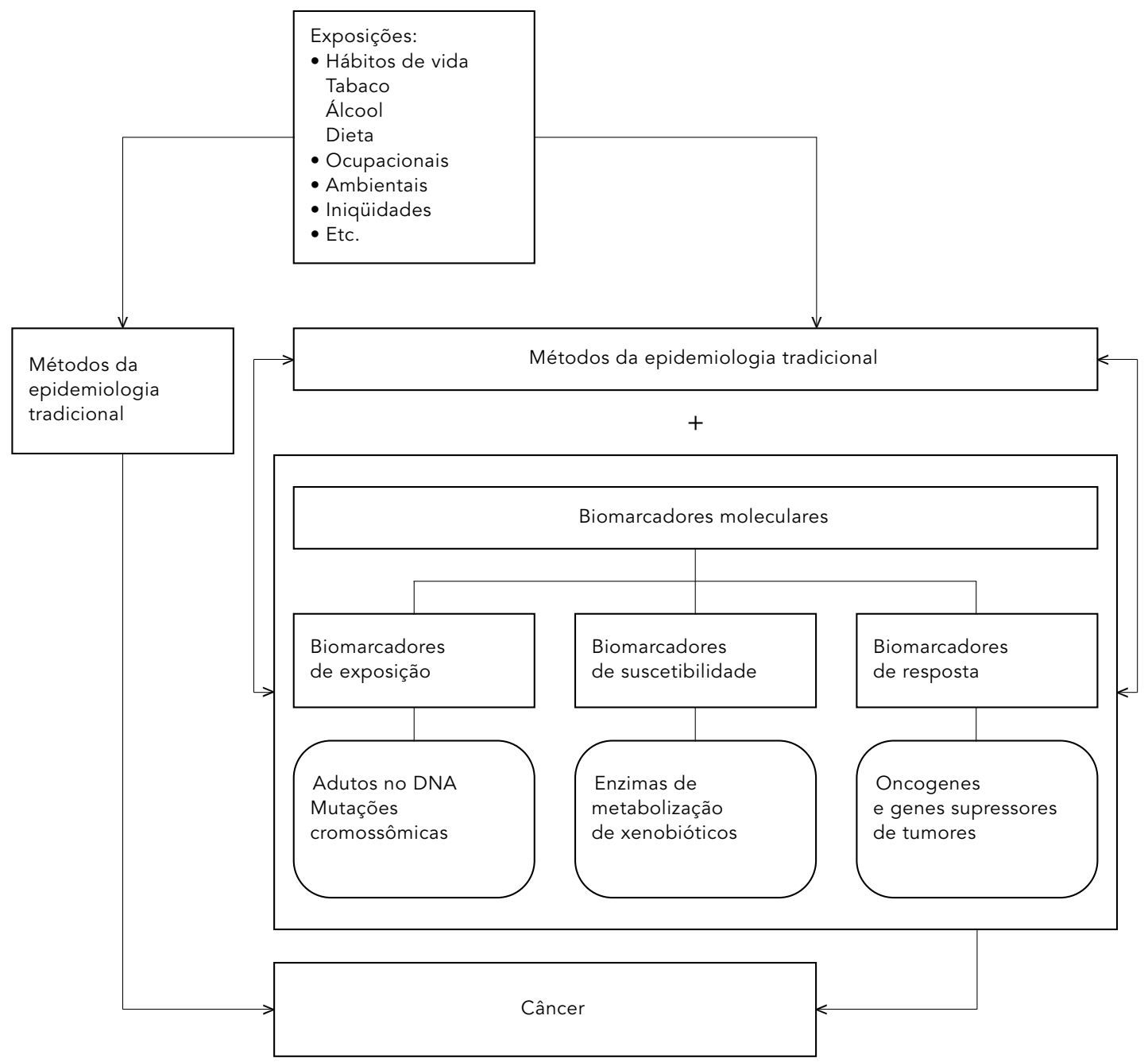

que visam avaliar a incidência de mutações cromossômicas em populações expostas, comparadas a grupos-controle. Entre os testes com aplicação no homem, destacam-se a análise citogenética de linfócitos periféricos e a pesquisa de micronúcleos em linfócitos ou em epitélios de descamação (Carrano \& Natarajan, 1988; Fenech et al., 1999). As mutações ou aberrações cromossômicas envolvem modificações na estrutura e no número de cromossomos, que podem ser identificadas ao microscópio ótico. Esse teste é considerado um indicador de genotoxicidade extremamente sensível (Hagmar et al., 1994). Pesquisas epidemiológicas recentes confirmaram uma maior inci- dência de câncer em indivíduos que apresentavam, cerca de 15 anos antes do aparecimento da doença, aumento na freqüência de aberrações cromossômicas em linfócitos, indicando haver efeito mutagênico semelhante no tecido alvo (Hagmar et al., 1998). Todavia, os autores não conseguiram estabelecer a contribuição efetiva de diferentes hábitos de vida e da ocupação no aumento da freqüência de aberrações cromossômicas e do conseqüente aparecimento de câncer.

Exposições ocupacionais, ingestão de álcool, tabagismo e dieta parecem influir na incidência de aberrações cromossômicas (Barale et al., 1998; Gonsebatt et al., 1997; Hagmar et 
al., 1994). O consumo crônico de bebidas alcoólicas tem sido relacionado a diferentes tipos de câncer (Jensen et al., 1996) e com o aumento, estatisticamente significante, na freqüência de linfócitos com aberrações cromossômicas numéricas e estruturais (Obe \& Anderson, 1987). Isso ocorre mesmo após longos períodos de abstinência sugerindo uma ação do álcool na capacidade de reparo celular e nas funções oxidativas da célula (Gattás \& Saldanha, 1997; Matsushima, 1987).

Os efeitos mutagênicos resultantes do uso de tabaco apresentam resultados contraditórios. Embora alguns autores tenham observado aumento na freqüência de aberrações cromossômicas estruturais em fumantes, resultados negativos também foram documentados (Au et al., 1998; Perera et al., 1987; Reuterwall, 1990). A técnica de citogenética com hibridização in situ por fluorescência, FISH (Fluorescence In Situ Hybridization), vem se mostrando útil na detecção de aberrações cromossômicas que ocorrem em baixa freqüência. Por meio dessa técnica de citogenética molecular observou-se aumento significativo na freqüência de translocações cromossômicas em fumantes (Conforti-Froes et al., 1998; Van Diemen et al., 1995).

Outro teste que também avalia alterações citogenéticas é o teste do micronúcleo (MN). Rápido e econômico, vem sendo aplicado para a avaliação de populações humanas expostas a agentes mutagênicos e carcinogênicos (Heddle et al., 1991). Os MN são identificados na interfase de células em divisão e se apresentam na forma de pequenos corpúsculos citoplasmáticos. Correspondem a fragmentos acêntricos resultantes de deleções cromossômicas ou cromossomos inteiros que se atrasam durante a anáfase da divisão celular. O exame de MN em células epiteliais, primeiramente descrito por Stich \& Rosin (1984), permite a observação direta do tecido alvo, por exemplo da boca, e relaciona-se à exposição ao álcool, tabagismo e aditivos alimentares, entre outros (Fenech et al., 1999; Ramirez et al., 1999).

\section{Biomarcadores de suscetibilidade}

Para dado nível de exposição a um carcinógeno, somente parte dos indivíduos expostos desenvolverá câncer. A maior parte das substâncias potencialmente carcinogênicas requerem ativação metabólica no organismo antes de se tornarem efetivamente cancerígenas. A suscetibilidade individual ao câncer parece depender, em parte, da capacidade, determinada geneticamente, de metabolizar e eliminar essas substâncias do organismo de forma eficiente.
Evidências apontadas em diferentes estudos indicam que inúmeros sistemas genéticos de controle e modulação do metabolismo enzimático de xenobióticos parecem estar envolvidos na gênese de diferentes tipos de câncer (d'Errico et al., 1999; Taningher et al., 1999). Os polimorfismos metabólicos que têm sido associados de forma mais consistente ao aumento do risco de câncer incluem o citocromo P450 (Kato et al., 1994), a glutationa S-transferase e a N-acetil-transferase (Antilla et al., 1995; Hirnoven et al., 1993).

As reações catalisadas por essas enzimas são divididas basicamente em duas fases. As enzimas da família do citocromo P450 (CYP) são classificadas como da fase I de metabolização e parecem estar diretamente relacionadas ao processo de ativação de grande parte dos xenobióticos. Na fase II, ocorre a transformação dos xenobióticos em produtos hidrossolúveis e de fácil excreção, principalmente pela ação das enzimas glutationa-S-transferase (GST) e N-acetil-transferase (NAT) (Awasthi et al., 1994).

Diferenças na freqüência de determinados alelos enzimáticos foram observadas entre populações de origens étnicas diversas (Arruda et al., 1998; Lin et al., 1994; Stephens et al., 1994). Um exemplo é verificado na enzima CYP2D6. Enquanto a freqüência de indivíduos pobres metabolizadores situa-se entre $5 \%$ e $7 \%$ em populações caucasóides, nos chineses corresponde somente a $1 \%$ da população (Wolf \& Smith, 1999). Diferenças semelhantes explicariam, em parte, a incidência maior ou menor de determinados tumores em diferentes grupos populacionais.

O polimorfismo de um ou mais genes que codificam para estas enzimas levam ao aumento da ativação de carcinógenos ou diminuem a capacidade de inativá-los (ou ambas situações), e é provável que indivíduos portadores desses polimorfismos tenham risco aumentado de câncer quando expostos a cancerígenos. O gene CYP1A1 é de crítica importância para o metabolismo do benzopireno, um hidrocarboneto policíclico aromático. Polimorfismos deste gene têm sido associados a cânceres de pulmão, esôfago, cabeça e pescoço (Kawajiri, 1999). No Brasil, Sugimura et al. (1994), em estudo na população do Rio de Janeiro, não identificaram relação entre polimorfismo do gene $C Y P 1 A 1 \mathrm{e}$ carcinoma broncogênico. Em publicação posterior, os autores relataram um outro polimorfismo do mesmo gene, conhecido como IleVal, devido à substituição da isoleucina por valina, o qual estaria associado a câncer de pulmão (Hamada et al., 1995). 
A redução na atividade das GST altera a detoxificação dos HPA e tem sido associada ao câncer de pulmão (Seidegard et al., 1986). Estudo de meta-análise que combinou dados de 12 estudos caso-controle, a deleção de GSTM1 foi associada com aumento de $40 \%$ no risco de câncer de pulmão (McWilliams et al., 1995). Também foi observado o efeito modificador das mutações GSTM1 e NAT2 na associação entre asbesto e mesotelioma (Hirnoven et al., 1995).

As enzimas NAT estão envolvidas nas reações de ativação e inativação de numerosos xenobióticos. Nos humanos, os genes NAT1 e NAT2 são responsáveis pela atividade da $\mathrm{N}$-acetil-transferase. Alelos polimórficos dos NAT1 e NAT2 foram detectados e poderiam estar modificando a suscetibilidade individual ao câncer (Hirnoven, 1999). O nível de atividade das NAT2 determina as taxas de detoxificação ou ativação das aminas aromáticas. Ensaios para tipagem de fenótipos ou genótipos têm sido usados para classificar indivíduos em acetiladores rápidos ou lentos (Ambrosone et al., 1996). Um estudo genético examinou três polimorfismos do NAT2 responsáveis por cerca de $90 \%$ a $95 \%$ do fenótipo acetilador lento (com dois ou mais alelos mutantes) e indicou um aumento do risco de câncer entre mulheres na pós-menopausa, acetiladoras lentas e fumantes de vinte ou mais cigarros por dia, tanto entre as que tinham este padrão de consumo há dois ou há vinte anos. A análise por maços/ano indicou um risco aumentado de câncer de mama, cerca de três vezes, entre mulheres na pósmenopausa, acetiladoras lentas e fumantes de mais de 365 maços/ano de cigarros. Os autores do estudo observaram que, embora o risco de câncer de mama estivesse aumentado com o total de anos de tabagismo, a intensidade pareceu ser mais importante do que a duração para o risco (Ambrosone et al., 1996). Esse potencial efeito dos polimorfismos NAT como modificadores de respostas individuais à exposição a agentes ambientais foi também mostrado por outros dois estudos. O genótipo NAT2, acetilador lento, foi relacionado com o aumento do risco de mesotelioma (Hirnoven et al., 1996), e o genótipo NAT1, com alta atividade, aumentou o risco de câncer de pulmão relacionado ao tabaco (Bouchardy et al., 1998). Portanto, as evidências do efeito dos polimorfismos NAT são contraditórias e não permitem conclusões definitivas sobre o seu papel na gênese do câncer (Hirnoven, 1999).

Os dados disponíveis em muitas situações levam a interpretações confusas. Um exemplo é dado pela hipótese de uma relação entre o controle genético do metabolismo da debriso- quina, um agente anti-hipertensivo, e o risco de câncer de pulmão. A enzima CYP2D6 metaboliza esta e outras substâncias. Sua atividade é determinada pela razão de metabolização: administração de debrisoquina e excreção de 4-hidroxi-debrisoquina pela urina. Os indivíduos são classificados em dois fenótipos básicos: grandes (extensive metabolizers) ou pobres metabolizadores (Wolf \& Smith, 1999). Os primeiros estudos do efeito da metabolização da debrisoquina revelaram risco seis vezes maior de câncer de pulmão entre os indivíduos classificados como grandes metabolizadores, quando comparados a indivíduos pobres metabolizadores (Ayesh et al., 1984; Caporaso et al., 1990). Estudos subseqüentes sobre este tópico revelaram riscos mais modestos (Amos et al., 1992; Wolf et al., 1992) ou ausência de evidências do excesso de risco de câncer de pulmão relacionado ao genótipo responsável pelo metabolismo da debrisoquina (Shaw et al., 1995). Esses resultados contraditórios dificultam aceitar a participação do polimorfismo do gene CYP2D6 na causalidade do câncer de pulmão (Wolf \& Smith, 1999).

As variações detectadas nos riscos obtidos em diferentes estudos de polimorfismos genéticos podem ser a expressão de fatores como a prevalência do polimorfismo investigado, variações na acurácia dos métodos laboratoriais empregados e tamanho da população em estudo. Está bem estabelecido que vieses podem ser causados pelas variações da sensibilidade e especificidade do teste de acordo com a prevalência do fator de risco estudado, interferindo no cálculo do risco (Rothman et al., 1993). Em síntese, as evidências epidemiológicas dos efeitos dos polimorfismos de suscetibilidade podem, no atual estágio das pesquisas, ser aceitas apenas como sugestivas mas não como determinantes no processo de carcinogênese.

\section{Biomarcadores de efeito}

Na maioria dos cânceres, têm sido identificadas mutações em seqüências de DNA em loci cromossômicos específicos (Blot \& Fraumeni, 1996). As classes de lesões do DNA incluem basicamente as perdas ou deleções, substituição de pares de bases, inserções, amplificações, duplicações, inversões e translocações (Greenblatt et al., 1994). Não está claro se tais alterações são causa ou conseqüência de câncer. De fato, a história natural da doença parece envolver não uma, mas várias mutações genéticas.

O gene $p 53$ está localizado no braço curto do cromossomo 17 e codifica para uma proteína nuclear de 53.000 daltons, razão da designa- 
ção p53, com importante função reguladora do ciclo celular. As mutações no p53 são eventos genéticos freqüentemente observados em vários tipos de câncer em humanos e relacionados com o agente ambiental envolvido (Greenblatt et al., 1994). O processo de gênese tumoral está intimamente associado à reprodução celular, ou seja, aos mecanismos que regem o ciclo celular. Para que a cada ciclo duas células-filhas idênticas sejam geradas, é necessário que ocorra duplicação e subseqüente segregação dos cromossomos e demais componentes da célula. Cromossomos são replicados numa fase conhecida como S (síntese) e segregados para as células-filhas na fase $\mathrm{M}$ (mitose) do ciclo celular. Esse processo ocorre de forma contínua, passando de G1-S-G2-M, sendo G1 e G2 (gap 1 e 2) fases que antecedem os períodos de síntese e divisão celular. O controle do ciclo celular é feito basicamente por intermédio de proteínas que atuam nas fases G1 e G2, acionando mecanismos de reparo ou interrompendo o processo de divisão celular quando detectam mutações no material genético. Grande parte dos genes supressores tumorais sintetiza proteínas com funções de regulação do ciclo celular, as quais, quando ausentes ou ineficientes, podem contribuir para a evolução de clones de células tumorais. O p53 é uma das proteínas que controla o ciclo celular, durante a fase G1, retardando o processo de divisão para que ocorra o reparo ou mesmo impedindo a divisão celular através de apoptose (morte celular). Por outro lado, mutações no gene $p 53$ induzem à formação de proteínas alteradas que não conseguem interromper o processo de divisão celular e, sem tempo suficiente para que ocorra o reparo do DNA, a célula carrega o dano para as divisões subseqüentes possibilitando a formação dos tumores (Harris, 1993).

A análise do espectro de mutações no $p 53$ em determinada exposição ambiental pode ser útil para identificar fatores envolvidos na etiopatogênese do câncer. Mutações do p53 no câncer de pulmão foram relacionadas com exposições ocupacionais nas indústrias petroquímica e metalúrgica e na exposição ao níquel (Harty et al., 1996). A prevalência de mutações do gene $p 53$ nos tecidos neoplásicos varia de acordo com o sítio anatômico do tumor, de $0 \%$, em tumores de testículos e da hipófise, a mais de $50 \%$, nos tumores de pulmão e cólon (Greenblatt et al., 1994).

Além de mutações no $p 53$, presente em cerca de $20 \%$ dos tumores de mama, alterações em outros dois genes supressores de tumor, $B R C A 1$ e $B R C A 2$, têm sido identificadas e conferem aumento da suscetibilidade à doença nas mulheres de famílias portadoras, de acordo com um padrão mendeliano de hereditariedade (Moll et al., 1992). Mulheres portadoras de mutação herdada BRCA1 têm a suscetibilidade aumentada para o câncer de mama, e mais de $70 \%$ deverão desenvolver a doença até os setenta anos de idade. A presença de mutações no $p 53$ também é indicativo de pior prognóstico da doença (Perera, 1996).

Com relação ao proto-oncogene ras, a freqüência de mutações em diferentes tumores é da ordem de $20 \%$ a $35 \%$. A concepção atual é que as mutações do ras e do p53 são eventos comuns no câncer que nem sempre ocorrem independentemente (Greenblatt et al., 1994). Os pacientes portadores de câncer de pulmão com mutações ras têm sobrevida menor do que aqueles com ausência da mutação (Rodenhuis \& Slebos, 1990; Westra et al., 1993).

No atual estágio de conhecimento dos mecanismos celulares do câncer de pulmão, câncer dos mais estudados na sua dinâmica molecular, sugere-se que as mutações ras estariam mais ligadas ao adenocarcinoma, tipo histológico com vínculo menos intenso com o tabaco. Já os polimorfismos CYP e GST parecem ter maior prevalência em tumores epidermóides, tipo histológico mais relacionado ao tabagismo (Caporaso \& Landi, 1994; Slebos et al., 1991). Entretanto, separações rígidas são pouco produtivas, pois alguns estudos têm mostrado que mutações ras também parecem ser diretamente causadas pela exposição aos carcinógenos presentes no tabaco (Rodenhuis \& Slebos, 1992), e já foram identificadas relações entre tabagismo, polimorfismos CYP1A1 e adenocarcinoma do pulmão (Nakachi et al., 1995).

\section{Aspectos éticos na epidemiologia genética e molecular}

Inúmeras questões éticas emergem nesse contexto de desenvolvimento da biologia molecular. Algumas dizem respeito à pesquisa epidemiológica per se e aos indivíduos envolvidos. Outras são mais amplas, pois repercutem na própria vida das pessoas (Hossne, 1998). Em relação ao primeiro grupo de questões, deve-se considerar que até o advento dos progressos recentes da biologia molecular, a pesquisa epidemiológica avaliava as exposições ambientais, restringindo-se, na prática, à aplicação de questionários e, em algumas poucas situações, à execução de avaliações ambientais. Com o crescente uso das técnicas moleculares nos estudos epidemiológicos, o que se busca agora é também obter mensurações de exposição no 
meio interno dos indivíduos - doses internas ou marcadores de suscetibilidade individual (Hunter \& Caporaso, 1997).

Uma vez definidos, tais biomarcadores poderiam ser utilizados, por exemplo, para a seleção de indivíduos no mercado de trabalho. Hipoteticamente, a disponibilidade dessa tecnologia poderia submeter os trabalhadores durante o processo de seleção a avaliações quanto à sua condição de portadores de determinados polimorfismos genéticos. Os resultados destes exames indicariam os mais aptos e menos propensos a desenvolver a doença quando expostos a cancerígenos específicos presentes no ambiente de trabalho. Obviamente, nessa situação, haveria um contingente de marginalizados do mercado de trabalho por sua incompetência genética herdada (Rothenberg et al., 1997; Soskolne, 1997).

O impacto social futuro desses novos conhecimentos é difícil de ser dimensionado, mas a sociedade deve buscar tanto desenvolver mecanismos de controle, quanto fomentar discussões sobre os aspectos éticos da utilização dessas novas técnicas e conhecimentos científicos. Um processo de consulta pública conduzida pelos institutos nacionais de saúde dos Estados Unidos concluiu que os representantes da população no parlamento têm poucas dificuldades para absorver os aspectos essenciais da ciência para a discussão de questões éticas em pesquisas com seres humanos (Anonymous, 2000). Nos países em desenvolvimento, esse cenário talvez seja um pouco diferente, mas a busca para tornar a população cientificamente mais educada deve fazer parte dos objetivos da construção de uma sociedade democrática.

\section{Conclusões}

A aplicação dos biomarcadores na epidemiologia deverá se acentuar nos próximos anos, embora inúmeras questões estejam ainda sem resposta, incluindo: problemas de mensuração de exposições mais remotas; dificuldades de saber o que o biomarcador está avaliando; probabilidade de ocorrência de confundimento em algumas situações; desconhecimento da relação entre processo da doença e o nível de biomarcadores; problemas de validade das mensurações laboratoriais; falta de clareza sobre a aplicação dos biomarcadores para as políticas de saúde pública (Pearce \& Boffetta, 1997).

O viés de confundimento é uma preocupação em todos os estudos epidemiológicos, incluindo aqueles que se utilizam de biomarcadores. Os biomarcadores de exposição podem ser importantes para o exame de potenciais vieses de confundimento. O erro de classificação de uma variável confundidora, entretanto, impede o controle de confundimento. Assim, em um estudo caso-controle com informações sobre exposição obtidas por questionários, os biomarcadores poderiam ser usados para validar as informações conseguidas em um subgrupo de participantes (casos e não-casos). Por outro lado, o uso de biomarcadores de exposição não reduz a necessidade de controle para a confusão de variáveis e, eventualmente, podem introduzir confundimento no estudo. Por exemplo, os trabalhadores em um estudo epidemiológico ocupacional podem ser classificados de acordo com os níveis de adutos HPADNA, mas isto irá indicar a exposição total de HPA de todas as fontes, inclusive tabagismo. Portanto, os efeitos dose-resposta poderão estar confundidos pelo consumo de cigarros, e o grupo classificado como com alta exposição aos HPA pode apresentar um maior risco de câncer de pulmão, mas não como decorrência da exposição aos HPA, mas sim por causa de outros carcinógenos presentes no tabaco (Pearce \& Boffetta, 1997).

Os biomarcadores de suscetibilidade permitem a oportunidade de identificar interações entre fatores genéticos e ambientais (Hirnoven et al., 1993; Jourenkova-Miranova et al., 1998). Além disso, definidos na cadeia causal, estes biomarcadores podem identificar nas populações os subgrupos com maiores riscos de desenvolver a doença, permitindo intervenções mais eficazes e mecanismos de avaliação mais adequados (McMichael, 1994).

Algumas mutações, como os genes BRCA1 e $B R C A 2$, têm importância limitada sobre o risco atribuível de câncer (significância preditiva) decorrente da baixa prevalência na população. Também os polimorfismos genéticos apresentam, em geral, prevalência restrita na população. A pequena fração atribuível de câncer decorrente destas alterações moleculares pode levar a conclusões com vieses. Diferenças na freqüência de polimorfismos têm sido detectadas entre diferentes grupos étnicos e populações, provavelmente como decorrência de exposições a carcinógenos específicos ou de características genéticas herdadas (Greenblatt et al., 1994). A melhor compreensão da associação entre câncer, exposições ambientais e biomarcadores de suscetibilidade em determinada região geográfica depende do conhecimento da distribuição na população de genes metabolizadores de xenobióticos (Conforti-Froes et al., 1998). Na cidade de São Paulo, os polimorfismos gênicos CYP2E1 e GSTM1 foram es- 
tudados em diferentes grupos populacionais e os resultados mostraram menor freqüência de deleção do gene GSTM1 em mulatos, quando comparados aos demais grupos (Gattás \& Soares-Vieira, 2000).

As epidemiologias molecular e genética não se emanciparão do modelo clássico da epidemiologia, pois a obtenção de conclusões válidas exige seguir princípios bem estabelecidos que regem a investigação epidemiológica: seleção apropriada de casos e não-casos, controle de variáveis de confundimento e adequado tamanho de amostras para detectar diferenças

\section{Agradecimentos}

Este trabalho foi conduzido dentro do conjunto de suporte financeiro proporcionado pela Comissão Européia (RC/98/013077), Fundação de Amparo à Pesquisa do Estado de São Paulo (Processo 97/7522-8) e Universidade de São Paulo (Processos 97.1.24410.1.0 e 99.1.17693.1.2) - VWF e Laboratório de Investigação Médica, Hospital das Clínicas, Faculdade de Medicina, Universidade de São Paulo - GJFG. importantes (Greenblatt et al., 1994; Vineis et al., 1999). Para que a história natural do câncer e de outras doenças seja melhor compreendida será necessária a aproximação de epidemiologistas e biólogos moleculares, procurando compatibilizar linguagens e métodos de pesquisa que são distintos. Nos países com liderança em pesquisa científica, essa aproximação já é realidade, e grupos híbridos trabalham ativamente em diversos projetos. No Brasil, as iniciativas de aproximação são ainda tímidas, mas seguir essa trajetória plena de dúvidas e desafios será inevitável.

\section{Referências}

AMBROSONE, C. B.; FREUDENHEIM, J. L.; GRAHAM, S.; MARSHALL, J. R.; VENA, J. R.; BRASURE, J. R.; MICHALEK, A. M.; LAUGHLIN, R.; NEMOTO, T.; GILLENWATER, K. A.; HARRINGTON, A. \& SHIELDS, P. G., 1996. Cigarette smoking, N-acetyltransferase 2 genetic polymorphisms, and breast cancer risk. JAMA, 276:1494-1501.

AMOS, C. T.; CAPORASO, N. \& WESTON, A., 1992. Host factors in lung cancer risk: A review of interdisciplinary studies. Cancer Epidemiology, Biomarkers \& Prevention, 1:505-513.

ANONYMOUS, 2000. Benefits of increased public participation. Nature, 405:259.

ANTILLA, S.; LUOSTARINEN, L.; HIRNOVEN, A.; ELOVAARA, E.; KARJALAINEN, A.; NURMINEN, T.; HAYES, J. D; VAINIO, H. \& KETTERER, B., 1995. Pulmonary expression of glutathione S-transferase M3 in lung cancer patients: Association with GSTM1 polymorphism, smoking and asbestos exposure. Cancer Research, 55:3305-3309.

ARRUDA, V. R.; GRIGNOLLI, C. E.; GONCCALVES, M. S.; SOARES, M. C.; MENEZES, R.; SAAD, S. T. O. \& COSTA, F. F., 1998. Prevalence of homozygosity for the deleted alleles of glutathione S-transferase mu (GSTM1) and theta (GSTT1) among distinct ethnic groups from Brazil: Relevance to environmental carcinogenesis? Clinical Genetics, 54:210214.

AU, W. W.; CAJAS-SALAZAR, N. \& SALAMA, S., 1998. Factors contributing to discrepancies in population monitoring studies. Mutation Research, 400: 467-478.

AWASTHI, Y. C.; SHARMA, R. \& SINGHAL, S. S., 1994. Human glutathione S-transferases: Minireview. International Journal of Biochemestry, 26:295-308. 
AYESH, R.; IDLE, J. R.; RITCHIE, L. C.; CROTHER, M. W. \& HETZEL, M. R., 1984. Metabolic oxidation phenotypes as markers for susceptibility to lung cancer. Nature, 312:169-170.

BARALE, R.; CHELOTTI, L.; DAVINI, T.; DELRY, S.; ANDREASSI, M. G.; BALLARDIN, M.; BULLERI, M.; HE, J.; BALDACCI, S.; DI PEDE, F; GEMIGNANI, F. \& LANDI, S., 1998. Sister chromatid exchange and micronucleus frequency in human lymphocytes of 1,650 subjects in an Italian population: II. Contribution of sex, age and lifestyle. Environmental Molecular Mutagenesis, 31:228242.

BLOT, W. J. \& FRAUMENI Jr., J. F., 1996. Cancers of lung and pleura. In: Cancer Epidemiology and Prevention (D. Schottenfeld \& J. F. Fraumeni Jr., ed.), pp. 637-665, 2nd Ed. New York: Oxford University Press.

BOUCHARDY, C.; MITRUNEN, K.; WIKMAN, H.; HUSGAFVEL-PURSIANINEN, K.; DAYER, P.; BENHAMOU, S. \& HIRNOVEN, A., 1998. N-acetiltransferase NAT1 and NAT2 genotypes and lung cancer risk. Pharmacogenetics, 8:191-198.

BROWNSON, R. C.; ALAVANJA, M. C.; CAPORASO, N.; BERGER, E. \& CHANG, J. C., 1997. Family history of cancer and risk of lung cancer in lifetime nonsmokers and long-term ex-smokers. International Journal of Epidemiology, 26:256-263.

CAPORASO, N. E. \& LANDI, M. T., 1994. Molecular epidemiology: A new perspective for the study of toxic exposures in man. A consideration of the influence of genetic susceptibility factors on risk in different lung cancer histologies. Medicina del Lavoro, 85:68-77.

CAPORASO, N. E.; TUCKER, M. A.; HOOVER, R. N.; HAYES, R. B.; PICKLE, L. W.; ISSAQ, H. J.; MUSDHIK, G. M.; GREEN-GALLO, L.; BUIVYS, D. \& AISNER, S., 1990. Lung cancer and the debrisoquine metabolic phenotype. Journal of the National Cancer Institute, 82:1264-1272.

CARRANO, A. V. \& NATARAJAN, A. T., 1988. International Commission for Protection against Environmental Mutagens and Carcinogens. ICPEMC publication no. 14. Considerations for population monitoring using cytogenetic techniques. Mutation Research, 204:379-406.

CONFORTI-FROES, N.; EL-ZEIN, R. \& AU, W., 1998. Genetic polymorphism and their contribution to cancer suscetibility. Cadernos de Saúde Pública, 14(Sup. 3):7-13

D'ERRICO, A.; MALATS, N.; VINEIS, P. \& BOFFETTA, P., 1999. Review of studies of selected metabolic polymorphisms and cancer. In: Metabolic Polymorphisms and Susceptibility to Cancer (P. Vineis, N. Malats, M. Lang, A. d'Errico, N. Caporaso, J. Cuzick \& P. Boffetta, ed.), pp. 323-394, Lyon: International Agency for Research on Cancer Press.

DAY, N. E. \& BROWN, C. C., 1980. Multistage models and primary prevention of cancer. Journal of the National Cancer Institute, 64:977-989.

DUNHAM, I.; SHIMIZU, N.; ROE, B. A.; CHISSOE, S.; HUNT, A. R.; COLLINS, J. E. et al., 1999. The DNA sequence of human chromosome 22. Nature, 402: 489-495.

ELLSWORTH, D. L.; HALLMAN, D. M. \& BOERWINKLE, E., 1997. Impact of the Human Genome Pro- ject on epidemiologic research. Epidemiologic Review, 19:3-13.

FENECH, M.; HOLLAND, N.; CHANG, W. P.; ZEIGER, E. \& BONASSI, S., 1999. The Human Micronucleus Project - An international collaborative study on the use of the micronucleus technique for measuring DNA damage in humans. Mutation Research, 428:271-283.

FLANDERS, W. D. \& KHOURY, M. J., 1996. Analysis of case-parental control studies: Method for the study of associations between disease and genetic markers. American Journal of Epidemiology, 144:696-703.

GATTÁS, G. J. F. \& SALDANHA, P. H., 1997. Chromosomal aberrations in peripheral lymphocytes of abstinent alcoholics. Alcoholism Clinical Experimental Research, 21:238-243.

GATTÁS, G. J. F. \& SOARES-VIEIRA, J. A., 2000. Cytochrome P450-2E1 (CYP2E1) and Glutathione STransferase mu (GSTM1) polymorphisms among Caucasians and Mulattoes from Brazil. Occupational Medicine, 50:508-511.

GAUDERMAN, W. J. \& MORRISON, J. L., 2000. Evidence for age-specific genetic relative risks in lung cancer. American Journal of Epidemiology, 151:41-49.

GONSEBATT, M. E.; VEGA, L.; SALAZAR, A. M.; MONTERO, R.; GUZMAN, P.; BLAS, J.; DEL RAZO, L. M.; GARCIA-VARGAS, G.; ALBORES, A.; CEBRIAN, M. E.; KELSH, M. \& OSTROSKY-WEGMAN, P., 1997. Cytogenetics effects in human exposure to arsenic. Mutation Research, 386:219-228.

GREENBLATT, M. S.; BENNET, W. P.; HOLLSTEIN, M. \& HARRIS, C. C., 1994. Mutations in p53 suppressor gene: Clues to cancer etiology and molecular pathogenesis. Cancer Research, 54:4855-4878.

HAGMAR, L.; BONASSI, S.; STRÖMBERG, U.; MIKOCZY, Z.; LANDO, C.; HANSTEEN, I.-L.; MONTAGUD, A. H.; KNUDSEN, L.; NORPPA, H.; REUTERWALL, C.; TINNERBERG, H.; BROGGER, A.; FORNI, A.; HÖGSTEDT, B.; LAMBERT, B.; MITELMAN, F.; NORDENSON, I.; SALOMAA, S. \& SKERFVING, S., 1998. Cancer predictive value of cytogenetic markers used in occupational health surveillance programs: A report from an ongoing study by the European Study Group on Cytogenetic Biomarkers and Health. Mutation Research, 405:171-178.

HAGMAR, L.; BROGGER, A.; HANSTEEN, I.-L.; HEIM, B.; HÖGSTEDT, B.; KNUDSEN, L.; LAMBERT, B.; LINNAINMAA, F; MITELMAN, F.; NORDENSON, I.; REUTERWALL, C.; SALOMAA, S.; SKERFVING, S. \& SORSA, M., 1994. Cancer risk in humans predicted by increased levels of chromosome aberrations in lymphocytes: Nordic study group on the health risk of chromosome damage. Cancer Research, 54:2919-2922.

HAMADA, G. S.; SUGIMURA, H.; SUZUKI, I.; NAGURA, K.; KOYOKAMA, E.; IWASE, T.; TANAKA, M.; TAKAHASHI, T.; WATANABE, S.; KONO, I. \& TSUGANE, S., 1995. The heme-binding region polymorphism of cytochrome P450lA1 (CyplA1), rather than the Rsal polymorphism of IIEl (CypIIE1), is associated with lung cancer in Rio de Janeiro. Cancer Epidemiology, Biomarkers \& Prevention, 4:63-67. 
HARRIS, C. C., 1987. Tobacco smoke and lung cancer: Who is susceptible? Annals of Internal Medicine, 105:607-609.

HARRIS, C. C., 1993. The p53 tumor suppressor gene: At the crossroads of molecular carcinogenesis, molecular epidemiology and cancer risk assessment. Science, 262:1980-1981.

HARTY, L. C.; GUINEE Jr., D. G.; TRAVIS, W. D.; BENNET, W. P.; JETT, J.; COLBY, T. V.; TAZELAAR, H.; TRASTEK, V.; PAIROLERO, P.; LIOTTA, L. A.; HARRIS, C. C. \& CAPORASO, N. E., 1996. p53 mutations and occupational exposures in a surgical series of lung cancers. Cancer Epidemiology, Biomarkers \& Prevention, 5:997-1003.

HEDDLE, J. A.; CIMINO, M. C.; HAYASHI, M.; ROMAGNA, F.; SHELBY, M. D.; TUCKER, J. D.; VANPARYS, P. H. \& MacGREGOR, J. T., 1991. Micronuclei as an index of cytogenetic damage: Past, present and future. Environmental and Molecular Mutagenesis, 18:277-291.

HEMMINKI, K. \& VAITTINEN, P., 1998. National database of familial cancer in Sweden. Genetic Epidemiology, 15:225-236.

HIRNOVEN, A., 1999. Polymorphic NATs and cancer predisposition. In: Metabolic Polymorphisms and Susceptibility to Cancer (P. Vineis, N. Malats, M. Lang, A. d'Errico, N. Caporaso, J. Cuzick \& P. Boffetta, ed.), pp. 251-270, Lyon: International Agency for Research on Cancer Press.

HIRNOVEN, A.; HUSGAFVEL-PERSIAINEN, K.; ANTILLA, S. \& VAINIO, H., 1993. The GSTM1 null genotype as a potential risk modifier for squamous cell carcinoma of the lung. Carcinogenesis, 14:1479-1481.

HIRNOVEN, A.; PELIN, K.; TAMMILEHTO, L.; KARJALAINEN, A.; MATTSON, K. \& LINNAINMAA, K., 1995. Inherited GSTM1 and NAT2 defects as concurrent risk modifiers for asbestos-associated human malignant mesothelioma. Cancer Research, 55:2981-2983.

HIRNOVEN, A.; SAARIKOSKI, S.; LINNAINMAA, K.; KOSKINEN, K.; HUSGAFVEL-PERSIAINEN, K. \& VAINIO, H., 1996. GST and NAT genotypes and asbestos-associated pulmonary disorders. Journal of the National Cancer Institute, 88:18531856.

HOSSNE, W. S., 1998. Epidemiologia, desenvolvimento tecnológico e ética. In: Epidemiologia: Contextos e Pluralidade (R. P. Veras, M. L. Barreto, N. Almeida Filho \& R. B. Barata, org.), pp. 147-166, Rio de Janeiro: Editora Fiocruz/ABRASCO.

HUNTER, H. \& CAPORASO, N., 1997. Informed consent in epidemiologic studies involving genetic markers. Epidemiology, 8:596-599.

JENSEN, O. M.; PAINE, S. L.; McMICHAEL, A. J. \& EWERTZ, M., 1996. Alcohol. In: Cancer Epidemiology and Prevention (D. Schottenfeld \& J. F. Fraumeni Jr., ed.), pp. 291-318, 2nd Ed. New York: Oxford University Press.

JOURENKOVA-MIRANOVA, N.; WIKMAN, H., BOUCHARDY, C.; VOHO, A.; DAYER, P.; BENHAMOU, S. \& HIRNOVEN, A., 1998. Role of glutathione Stransferase GSTM1, GSTM3, GSTP1 and GSTT1 genotypes in modulating susceptibility to smoking-related lung cancer. Pharmacogenetics, 8:495502
KATO, S.; SHIELDS, P. G.; CAPORASO, N. E.; SUGIMURA, H.; TRIVERS, G. E.; TUCKER, M. A.; TRUMP B. F.; WESTON, A. \& HARRIS, C. C., 1994. Analysis of cytocrome $\mathrm{P} 450$ 2E1 genetic polymorphisms in relation to human lung cancer. Cancer Epidemiology, Biomark \& Prevention, 3:515-518.

KAWAJIRI, K., 1999. CYP1A1. In: Metabolic Polymorphisms and Susceptibility to Cancer (P. Vineis, N. Malats, M. Lang, A. d'Errico, N. Caporaso, J. Cuzick \& P. Boffetta, ed.), pp. 159-171, Lyon: International Agency for Research on Cancer Press.

KHOURY, M. J., 1998. Genetic epidemiology. In: Modern Epidemiology (K. J. Rothman \& S. Greenland, ed.), pp. 609-621, Philadelphia: Lippincott, Williams and Wilkins.

KULLER, L. H., 1999. Invited commentary: Circular epidemiology. American Journal of Epidemiology, 9:897-903.

LIN, H. J.; HAN, C. Y.; BERNSTEIN, D. A.; HSIAO, W. LIN, B. K. \& HARDY, S., 1994. Ethnic distribution of the glutathione S-transferase mu-1 (GSTM1) null genotype in 1473 individuals and application to bladder cancer susceptibility. Carcinogenesis, 15:1077-1081.

MATSUSHIMA, Y., 1987. Chromosomal aberrations in the lymphocytes of alcoholics and former alcoholics. Neuropsychobiology, 17:24-29.

McMICHAEL, A. J., 1994. Molecular epidemiology: new pathway or new travelling companion? American Journal of Epidemiology, 140:1-11.

McWILLIAMS, J. E.; SANDERSON, B. S.; HARRIS, E. L.; RICHERT-BOE, K. E. \& HENNER, W. D., 1995. Glutathione S-transferase M1 (GSTM1) deficiency and lung cancer risk. Cancer Epidemiology, Biomarkers \& Prevention, 4:589-594.

MOLL, U.; RIOU, G. \& LEVINE, A. J., 1992. Two distinct mechanisms alter p53 in breast cancer: mutation and nuclear exclusion. Proceedings of the National Academy of Sciences of the United States of America, 89:7262-7266.

MONTESANO, R., 1990. Approaches to detect individual exposure to carcinogens. In: Complex Mix tures and Cancer Risk (H. Vainio, M. Sorsa \& A. J. McMichael, ed.), pp. 11-19, Lyon: International Agency for Research on Cancer Press.

MULVIHILL, J. J. \& TULINIUS, H., 1987. Cancer ecogenetics: Studying genetic and environment interactions through epidemiology. International Journal of Epidemiology, 16:337-340.

NAKACHI, K.; HAYASHI, S.; KAWAJIRI, K. \& IMAI, K. 1995. Association of cigarette smoking and CYP1A1 polymorphisms with adenocarcinoma of the lung by grades of differentiation. Carcinogenesis, 16 : 2209-2213.

OBE, G. \& ANDERSON, D., 1987. International commission for protection against environmental mutagens and carcinogens: Genetic effects of ethanol. Mutation Research, 186:177-200.

PEARCE, N. \& BOFFETTA, P., 1997. General issues of study design and analysis in the use of biomarkers in cancer epidemiology. In: Application of Biomarkers in Cancer Epidemiology (P. Toniolo, P. Boffetta, D. E. G. Shuker, N. Rothman, B. Hulka \& N. Pearce, ed.), pp. 47-57, Lyon: International Agency for Research on Cancer Press.

PERERA, F. P., 1996. Molecular epidemiology: Insights 
into cancer susceptibility, risk assessment, and prevention. Journal of the National Cancer Institute, 8:496-509.

PERERA, F. P.; HEMMINKI, K. H.; GRYZBOWSKA, E.; MOTYKIEWICZ, G.; MICHALSKA, J.; SANTELLA, R. M.; YOUNG, T. L.; DICKEY, C.; BRANDT-RAUF, P.; DE VIVO, I.; BLANER, W.; TSAI, W. Y. \& CHORAZY, M., 1992. Molecular and genetic damage in humans from environmental pollution in Poland. Nature, 360:256-258.

PERERA, F. P.; HEMMINKI, K. H.; YOUNG, T. L.; BRENNER, D.; KELLY, G. \& SANTELLA, R. M., 1988. Detection of polycyclic aromatic hydrocarbon-DNA adducts in white blood cells of foundry workers. Cancer Research, 48:2288-2291.

PERERA, F. P.; SANTELLA, R. M.; BRENNER, D.; POIRIER, M. C.; MUNSHI, A. A.; FISCHMAN, H. K. \& RYZIN, J. V., 1987. DNA adducts, protein adducts, and sister chromatid exchange cigarette smokers and nonsmokers. Journal of the National Cancer Institute, 79:449-456.

PHILLIPS, D. H.; HEWER, A.; MARTIN, C. N.; GARNER, R. C. \& KING, M. M., 1988. Correlation of DNA adduct levels in human lung with cigarette smoking. Nature, 336:790-792.

PITOT, H. C., 1996. Stages in neoplastic development. In: Cancer Epidemiology and Prevention (D. Schottenfeld \& J. F. Fraumeni Jr., ed.), pp. 65-79, 2nd Ed. New York: Oxford University Press.

RAMIREZ, A.; GATTÁS, G. J. F.; CARVALHO, M. B.; RAPOPORT, A. \& SALDANHA, P. H., 1999. Clinical implications of micronuclei frequency as a biomonitor for alcoholic patients with oral carcinomas. In: Oral Oncology (A. K. Varma, ed.), v. 6, pp. 199-204, London: Macmillan.

REUTERWALL, C., 1990. A Nordic data base on somatic chromosome damage in humans. Nordic study group on the health risk of chromosome damage. Mutation Research, 241:325-337.

RODENHUIS, S. \& SLEBOS, R. J., 1990. The ras oncogenes in human lung cancer. American Review of Respiratory Diseases, 142:S27-S30.

RODENHUIS, S. \& SLEBOS, R. J., 1992. Clinical significance of ras oncogene activation in human lung cancer. Cancer Research, 52(Sup.):2665S-2669S.

ROTHENBERG, K.; FULLER, B.; ROTHSTEIN, M.; DUSTER, T.; ELLIS-KAHN, M.; CUNNINGHAM, R.; FINE, B.; HUDSON, K.; KING, M.; MURPHY, P.; SWEGOLD, G. \& COLLINS, F., 1997. Genetic information and the workplace: Legislative approaches and policy challenges. Science, 275:1755-1757.

ROTHMAN, N.; STEWART, W. F.; CAPORASO, N. E. \& HAYES, R. B., 1993. Misclassification of genetic susceptibility biomarkers: Implications for casecontrol studies and cross-population comparisons. Cancer Epidemiology, Biomarkers \& Prevention, 2:299-303.

SCHULTE, P. A. \& PERERA, F. P., 1993. Molecular Epidemiology: Principles and Pratices. New York: Academic Press.

SCHULTE, P. A. \& PERERA, F. P., 1997. Transitional studies. In: Application of Biomarkers in Cancer Epidemiology (P. Toniolo, P. Boffetta, D. E. G. Shuker, N. Rothman, B. Hulka \& N. Pearce, ed.), pp. 19-29, Lyon: International Agency for Research on Cancer Press.
SEIDEGARD, J.; PERO, R. W.; MILLER, D. G. \& BEATTIE, E. J., 1986. A glutathione transferase in human leukocytes as a marker for the susceptibility to lung cancer. Carcinogenesis, 7:751-753.

SELLERS, T. A.; BAILEY-WILSON, J.; ELSTON, R. C.; WILSON, A. F; ELSTON, G. Z.; OOI, W. L. \& ROTHSCHILD, H., 1990. Evidence for Mendelian inheritance in the pathogenesis of lung cancer. Journal of the National Cancer Institute, 82:1272-1279.

SHAW, G. L.; FALK, R. T.; DESLAURIERS, J.; FRAME, J. X.; NESBIT, L. C.; PASS, H. I.; ISSAQ, H. J.; HOOVER, R. N. \& TUCKER, M. A., 1995. Debrisoquine metabolism and lung cancer risk. Cancer Epidemiology, Biomarkers \& Prevention, 4:41-48.

SILVA, G. R., 1990. Avaliação e perspectivas da epidemiologia no Brasil. In: Epidemiologia e desigualdade social: Os desafios do final do século. 1o Congresso Brasileiro de Epidemiologia, Anais, pp. 108-139. Rio de Janeiro: ABRASCO.

SIMPSON, A., 1999. Um caminho competitivo para a descoberta de novos genes. Notícias FAPESP, 40: 2-6.

SKIPPER, P. L. \& TANNENBAUM, S. R., 1990. Protein adducts in the molecular dosimetry of chemical carcinogens. Carcinogenesis, 11:7-18.

SLEBOS, R. J.; HRUBAN, R. H.; DALESIO, O.; MOOI, W. J.; OFFERHAUS, G. J. \& RODENHUIS, S., 1991. Relationship between K-ras oncogene activation and smoking in adenocarcinoma of the human lung. Journal of the National Cancer Institute, 83: 1024-1027.

SOSKOLNE, C. L., 1997. Ethical, social, and legal issues surrounding studies of susceptible populations and individuals. Environmental Health Perspectives, 105(Sup. 4):837-841.

SQUIRE, J. A.; WHITMORE, G. F. \& PHILLIPS, R. A., 1998. Genetic basis of cancer. In: The Basic Sicence of Oncology (I. F. Tannock \& R. P. Hill, ed.), pp. 48-78, $3^{\text {rd }}$ Ed. New York: McGraw-Hill.

STEPHENS, E. A.; TAYLOR, J. A.; KAPLAN, N.; YANG, C. H.; HSIEH, L. L.; LUCIER, G. W. \& BELL, D. A., 1994. Ethnic variation in the CYP2E1 gene: Polymorphism analysis of 695 African-Americans, European-Americans and Taiwanese. Pharmacogenetics, 4:185-192.

STICH, H. F. \& ROSIN, M. P., 1984. Micronuclei in exfoliated human cells as a tool for studies in cancer risk and cancer intervention. Cancer Letters, 22:241-253.

SUGIMURA, H.; SUZUKI, I.; HAMADA, G. S.; IWASE, T.; TAKAHASHI, T.; NAGURA, K.; IWATA, H.; WATANABE, S.; KINO, I. \& TSUGANE, S., 1994. Cytochrome P-4501Al genotype in lung cancer patients and controls in Rio de Janeiro, Brazil. Cancer Epidemiology, Biomarkers \& Prevention, 3:145-148.

TANINGHER, M.; MALACARNE, D.; IZZOTTI, A.; UGOLINI, D. \& PARODI, S., 1999. Drug metabolism polymorphisms as modulators of cancer susceptibility. Mutation Research, 436:227-261.

TOKUHATA, G. K. \& LILIENFELD, A. M., 1963. Familial aggregation of lung cancer in humans. Journal of the National Cancer Institute, 30:289-312.

TONIOLO, P.; BOFFETTA, P.; SHUKER, D. E. G.; ROTHMAN, N.; HULKA, B. \& PEARCE, N., 1997. Application of Biomarkers in Cancer Epidemiology. 
Workshop Report. Lyon: International Agency for Research on Cancer Press.

VAINIO, H.; MAGGE, P.; McGREGGOR, D. \& Mc MICHAEL, A. J., 1992. Mechanisms of Carcinogenesis in Risk Identification. Consensus Report. Scientific Publications No. 116. Lyon: International Agency for Research on Cancer Press.

VAN DIEMEN, P. C.; MAASDAM, D.; VERMEULEN, S . DARROUDI, F. \& NATARAJAN, A. T., 1995. Influence of smoking habits on the frequencies of structural and numerical chromosomal aberrations in human peripheral blood lymphocytes using the fluorescence in situ hybridization (FISH) technique. Mutagenesis, 10:487-495.

VINEIS, P., 1998. Interazione tra genetica e ambiente. Medicina del Lavoro, 89:117-123.

VINEIS, P.; D'ERRICO, A.; MALATS, N. \& BOFFETTA, P., 1999. Overall evaluation and research perspectives. In: Metabolic Polymorphisms and Susceptibility to Cancer (P. Vineis, N. Malats, M. Lang, A. d'Errico, N. Caporaso, J. Cuzick \& P. Boffetta, ed.), pp. 403-408, Lyon: International Agency for Research on Cancer Press.

VOGELSTEIN, B. \& KINZLER, K. W., 1998. The Genetic Basis of Human Cancer. New York: McGraw-Hill.

WESTRA, W. H.; SLEBOS, R. J.; OFFERHAUS, G. J.; GOODMAN, S. N.; EVERS, S. G.; KENSLER, T. W.; ASKIN, F. B.; RODENHUIS, S. \& HRUBAN, R. H., 1993. K-ras oncogene activation in lung adenocarcinomas from former smokers. Evidence that $\mathrm{K}$-ras mutations are an early and irreversible event in the development of adenocarcinoma of the lung. Cancer, 72:432-438.
WILD, C. P. \& PISANI, P., 1997. Carcinogen-DNA and carcinogen-protein adducts in molecular epidemiology. In: Application of Biomarkers in Cancer Epidemiology (J. Toniolo, P. Boffetta, D. E. G. Shuker, N. Rothman, B. Hulka \& N. Pearce, ed.), pp. 143-158, Lyon: International Agency for Research on Cancer Press.

WOLF, C. R. \& SMITH, G., 1999. Cytochrome P450 CYP2D6. In: Metabolic Polymorphisms and Susceptibility to Cancer (P. Vineis, N. Malats, M. Lang, A. d'Errico, N. Caporaso, J. Cuzick \& P. Boffetta, ed.), pp. 209-229, Lyon: International Agency for Research on Cancer Press.

WOLF, C. R.; SMITH, C. A. D.; COUGH, A. C.; MOSS, L. E.; VALLIS, K. A.; HOWARD, G.; CAREY, F. L.; MILLS, K.; McNEE, W.; CARMICHAEL, J. \& SPURR, N. K., 1992. Relationship between the debrisoquine hydroxylase polymorphism and cancer susceptibility. Carcinogenesis, 13:1035-1038.

WÜNSCH FILHO, V.; MAGALDI, C.; NAKAO, N. \& MONCAU, J. E. C., 1995. Trabalho industrial e câncer de pulmão. Revista de Saúde Pública, 29: 166-176.

WYNDER, E. L., 1990. Epidemiological issues in weak associations. International Journal of Epidemiology, 19(Sup. 1):S5-S7.

WYSZYNSKI, D. F., 1998. La epidemiología genética: Disciplina científica em expansión. Revista Panamericana de Salud Pública, 3:1.

YANG, Q.; KHOURY, M. J.; SUN, F. \& FLANDERS, W. D., 1999. Case-only design to measure gene-gene interaction. Epidemiology, 10:167-170. 